\title{
8 Das Metaformat der Subjektivität des Wesens
}

Der eben explizierte Zusammenhang von Sein und Wesen im Übergang von der Seins- zur Wesenslogik weist auch noch in anderer Weise auf die zu Beginn der Wesenslogik explizierte kategoriale Minimalstruktur von „Realsein überhaupt“: indem der Zusammenhang des Übergangs vom Sein zum Wesen auch ein bestimmtes inhaltliches Moment dieser kategorialen Minimalstruktur bereits verwirklicht, wenn auch noch nicht erläuternd entfaltet. Denn indem das Wesen als Logik „absoluter Relationalität“61 und begrifflicher Vermittlung nichts anderes gegenüber der unvermittelten, absolut äußerlichen Unmittelbarkeit des Seins ist, sondern vielmehr dieses Sein an sich selbst in dieses Wesen übergeht, beginnt die Wesenslogik bereits strukturell mit der Einsicht, dass die Äußerlichkeit des Seienden nichts bloß Vorgängiges und die Immanenz des Begrifflichen nichts bloß Nachträgliches gegeneinander sein dürfen. Indem das Wesen in obigem Zitat bereits gleich zu Anfang als Struktur innerer Unterschiede gefasst wird, deren Gegensatz kein Übergehen in Anderes mehr meint, kommt die kategoriale Prämisse zum Ausdruck, dass der realitätskategorisch fundamentale Unterschied von äußerlichem Vorhandensein (Sein) und begrifflich-vermittelnder Konstruktion (Wesen) nur mehr als innere Selbstunterscheidung eines Zusammenhangs des Realen zu denken ist. Das Wesen als die „absolute Gleichgültigkeit gegen die Grenze“ (GW 11, S. 243) und damit als „[F]reimachen von aller seienden und unmittelbaren Bestimmtheit“62 erlaubt es nicht mehr, auch letztgültige Unterscheidungen wie Unmittelbarkeit und Vermittlung, Gegebenheit und Konstruktion, Bestimmtsein und Bestimmen als einzig in äußerlichen Entgegensetzungen begriffene und sich so einseitig bloß begrenzende Bestimmungen zu verstehen: weil sie stets als Sich-von-sich-Unterscheiden oder als „absolute[r] Unters c h i e d " (GW 11, S. 262) des Wesens selbst gefasst sind. Ein weiterer wesentlicher Gesichtspunkt der Wesenslogik kommt hier ins Spiel: der Umstand, dass alle Bestimmungen des Seins in ihr erstmals aus der Metaperspektive des Subjektformats reformuliert werden, d.h. ihre primäre Perspektive sachhaltiger Unterschiede erfährt eine zusätzliche Codierung durch die Form der Selbstheit, in Bezug auf welche diese Unterschiede verstanden werden. Damit kommt erst in der Wesenslogik und der mit ihr verbundenen Grammatik der Bestimmbarkeit Hegels systematische Grundidee seit der Phänomenologie, die "Substanz als Subject“ (GW 9, S. 400) zu rekonzeptualisieren, erstmals explizit zum Tragen, wobei der strukturelle Subjektcharakter eben vor allem darin liegt, das logische Gesamtbild

61 Vgl. grundlegend Iber: Metaphysik absoluter Relationalität.

62 Iber: Metaphysik absoluter Relationalität, S. 49.

○ OpenAccess. (C) 2021 Claudia Wirsing, publiziert von De Gruyter. (cc)B By unter einer Creative Commons Namensnennung 4.0 International Lizenz.

Dieses Werk ist lizenziert 
der Wirklichkeit als Prozess der „Selbstrealisation“63 der Substanz des Wirklichen zu begreifen. „Selbstrealisation“ aber muss im Kontext unserer Frage so verstanden werden, dass damit keineswegs eine Art Konstruktivismus der Wirklichkeit nach der Maßgabe eines absoluten oder empirischen Ich gemeint wäre: Damit würde man eben den Grundimpuls Hegels komplett ignorieren und seine Rede vom Subjekt als absolute Form zur Tätigkeit eines Ich degradieren.Vielmehr wird mit der Idee der „Selbstrealisation“ der Substanz als Subjekt, also eines realen An-sich-Bestehenden (Substanz), das sowohl aus sich selbst und nur in der Selbstbeziehung verständlich, als auch in seinem Ansichsein nur in der Form dieser Verständlichkeit gegeben sein kann, die realitätskategoriale Grundforderung der Logik nochmals benennbar: Der Unterschied des äußerlichen Gegebenseins des Realen als Substanz und der begrifflichen Rekonstruktion intentionaler Gehalte als Subjekt ist keine letztbegründende Differenz äußerlicher Relata, sondern selbst nur abgeleitet aus einer zugrunde liegenden Struktur des „Realen überhaupt“, in der das An-sich-Bestehende immer schon einzig in der Weise begrifflicher Selbstverständlichkeit, d.h. als begrifflicher Aus-sich- und Durch-sich-Erschließungszusammenhang, zu begreifen ist. Die kategoriale Grundform des „Realen überhaupt“ muss nach dem Modell struktureller Subjektivität, wie es sich dann in der „Idee“ vollständig ausbildet, als Identität von Allgemeinheit (Identität) und Besonderheit (Bestimmtheit in Gegensätzen), und d.h. als vorgängiges ontologisches wie epistemologisches Verschränktsein scheinbar letztgültiger bestimmter Gegensätze wie der von Geist und Welt, Begriff und Realität, Denken und Sein, verstanden werden. Die Substanz, die als Subjekt gänzlich aus sich heraus verständlich und nur in der logischen Beziehung des Sich als immer schon vorgängige Erschlossenheit für das Begreifen begreifbar ist, kann so nicht anders als wesentlich von begrifflicher Natur sein. Selbstrealisation als strukturelles Subjekt meint dementsprechend in unserer Hinsicht vor allem, einen Raum des Begrifflichen als eine logische Sphäre den Unterschieden von Denken und Welt, Begriff und Realität vorzuordnen. Denn subjektformativ kann das Ansich-Bestehende wie gesehen nur darin sein, die vollständige selbstbezügliche Verständlichkeit begrifflicher Erschließung zu realisieren und Realisierung einzig in dieser Zugänglichkeit zu vollziehen: nicht aber darin, bloßes Objekt einer konstruierenden Ich-Intentionalität zu sein.

Aus dieser subjektartigen Neuformatierung des Bestimmungszusammenhangs leiten sich also, zusammengefasst, strukturell-funktional mindestens zwei Forderungen an die Grammatik der Wesensbestimmungen ab: Zum einen muss das Wesen aus sich selbst entwicklungsfähig sowie „rein aus sich selbst heraus

63 Henrich: Hegels Logik der Reflexion, S. 95. 
verständlich“64 sein. Als „Absolutes“ darf es nicht durch eine ihm äußerliche Reflexion als „Product, ein gemachtes“ (GW 11, S. 242) erscheinen, sondern muss das, was es ist, durch sich selbst sein: Also darf es nicht auf Voraussetzungen basieren, die ihm als nicht subjekthaft prozessierbare äußerlich bloß vorgegeben sind und damit nicht in der freien Selbstbeziehung einer Subjektstruktur generiert werden können. Zum anderen darf dem Wesen das Bestimmtsein überhaupt nicht verloren gehen, sondern muss ihm vielmehr in einer anderen Form als dem Sein, nämlich als Selbstbestimmung, zukommen. Der Ausdruck für die neue Gesamtformation des Wesens, welcher diese Forderungen zusammenbringt, ist „An und-Fürsichseyn“, das Hegel als „absolutes Ansichseyn“ (GW 11, S. 242) begreift. Dergestalt ist das Wesen die „Wahrheit des Seyn s “ (GW 11, S. 241): Denn erst mit dieser subjektförmigen Ebene der Selbstbeschreibung geht das, was seinslogisch die Bestimmungen ausgemacht hat, ihnen aber als Wissen äußerlich blieb, auch in ihren Begriff ein. Das „Bewußtseyn über die Form der innern Selbstbewegung ihres Inhalts“ (GW 21, S. 37) als „Sichwissen“ (GW 21, S. 16) wird damit in den Beziehungen, die in die Bestimmungen eingehen, zur Beschreibung der Bestimmungen von sich selbst. Die Wesenslogik denkt also den generativen Charakter der Beziehungen zwischen den Kategorien als Raum ihres Bestimmtseins in die Selbstbeschreibung der Kategorien hinein. Den Wesensbestimmungen ist es immanent, sich wechselseitig in sich selbst durch sich selbst aktiv hervorzubringen und nicht nur, wie im Sein, passiv und äußerlich ineinander überführt $\mathrm{zu}$ werden. Damit wird ein Begriff des Wesens als autogenerative autonome Struktur gewonnen, die den logischen Zusammenhang folgerichtig als Format von Subjektivität weiterentwickelt, und es ist zugleich gerade diese Basis eines Begriffs autonomer Subjektivität, aufgrund derer Hegel einen logischen Begriff kategorialer Minimalbedingungen des Realen entwerfen kann, ohne diese auf Subjektivität - im Sinne eines absoluten Konstruktivismus - verkürzen zu müssen.

Indem das Wesen also wesentlich „Reflexion“ ist, ist es „die Einheit mit sich in diesem seinem Unterschiede von sich“ (GW 11, S. 242). Die Negativität im Verhältnis der Bestimmungen im Wesen zueinander wird über den noch zu erläuternden Begriff des „Scheins“ in der Form des Sich bestimmt: d. h. als reflexive Selbstbeziehung eines Ganzen, welches auch in den widersprüchlichsten Gedankenbestimmungen innerhalb der Identität eines Sich verbleibt, welches seine Entgegensetzungen als Unterschiede einer begrifflichen Substanz verständlich machen kann. Diese Matrix des Subjektformats verwirklicht sich dann konsequent bis in die Mikrostrukturen der Reflexionsbestimmungen hinein: in der Art

64 Henrich: Hegels Logik der Reflexion, S. 95. 
und Weise, wie sich der Unterschied von anderen Bestimmungen als begrifflichsemantische Selbstbeziehung von Gedankenbestimmungen konzeptualisieren lässt. Im Subjektformat der Wesensstruktur denkt Hegel das Immer-schon-ineinander-enthalten-Sein kategorialer Unterschiede in der Weise „objektiven“ Bewusstseins: „objektiv“ deshalb, weil die Wesenslogik als Gegengewicht zur Kritik der Seinslogik an ontologischer Äußerlichkeit eine Kritik der bloßen Innerlichkeit des Wesens liefert, wie sie im klassisch-philosophischen Denken als Unterschied von Wesentlichem/Unwesentlichem und nachfolgend v.a. im „Schein“ in der Form eines hinter den Erscheinungen der Realität verborgenen unsichtbaren Wesens vorliegt (vgl. GW 11, S. 245-249). Genau damit aber eignet sie sich erneut besonders dafür, das kategoriale Reale als intersubjektiv zugängliches und in dieser Hinsicht objektives „Vorhandensein überhaupt“ zu denken. Aus dieser Fundamentaltheorie von Realsein leiten sich unmittelbar Folgerungen für die Beschreibung der Wissens- und Darstellungsverhältnisse bezüglich des Realen $\mathrm{ab}$, die so gesehen logisch notwendig sind, d.h. insofern als alternativlos zu gelten haben, als sie allen möglichen Alternativbeschreibungen wie Idealismus/Realismus immer schon vorhergehen und zugrunde liegen. Dies gilt es im Folgenden weiter zu erläutern und anhand der Logik der Reflexionsformen aufzuzeigen. Dabei ist es vor allem der radikale und originäre Hegel'sche Ansatz, den es genauer zu erläutern gilt: wie nämlich aus einer äußersten Reduktion auf ein scheinbar bloß Gedankliches, nämlich aus der Fokussierung auf die innere logische Gestalt der „absoluten Negativität“ des Wesens, die gesamte Kernformation eines Begriffs des wirklichen Wesens und fernerhin damit eine begriffliche Matrix des „Realseins überhaupt“ gewonnen werden kann. Hegels Ansatz ist es in der Tat, vom reinen Gedanken einer sich auf sich beziehenden Negativität, d.h. von der Idee reiner struktureller Selbstbezüglichkeit einsichtig machen zu wollen, warum und in welcher Weise Sein und Wesen, Bestimmtsein und Bestimmen, Unmittelbarkeit und Vermittlung, Gedanke und Wirklichkeit immer schon notwendig als kategorial ineinander integriert zu bestimmen sind. 